\title{
GROWTH OF InSb ON GaAs SUBSTRATES USING InAISb BUFFERS FOR MAGNETIC FIELD SENSOR APPLICATIONS
}

R. M. Biefeld and J. D. Phillips, Sandia National Laboratories, Albuquerque, NM 87185-0601

\section{ABSTRACT}

We report the growth of InSb on GaAs using InAlSb buffers of high interest for magnetic field sensors. We have grown samples by metal-organic chemical vapor deposition consisting of $\sim 0.55 \mu \mathrm{m}$ thick InSb layers with resistive InAlSb buffers on GaAs substrates with measured electron mobilities of $\sim 40,000 \mathrm{~cm}^{2} / \mathrm{V}$.s. We have investigated the $\operatorname{In}_{1-\mathrm{x}} \mathrm{Al}_{\mathrm{x}} \mathrm{Sb}$ buffers for compositions $x \leq 0.22$ and have found that the best results are obtained near $x=0.12$ due to the tradeoff of buffer layer bandgap and lattice mismatch.

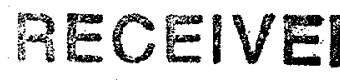

\section{INTRODUCTION}

Magnetic field sensors are becoming increasingly important for position sensing in computer controlled automotive applications, for which magnetoresistive sensors in particular are highly suited[1]. The sensitivity of magnetoresistors is highly dependent on the electron mobility, making high mobility InSb an attractive material choice. For device applications involving InSb, it is desirable to use wider bandgap substrates such as GaAs for reasons including electrical isolation at elevated temperatures, cost, and mechanical strength. High mobility InSb grown on GaAs has been demonstrated [2,3,4] and magnetoresistive sensors from these materials are currently in production[5]. It is desirable for these devices to reduce the film thickness in order to reduce power consumption and die size. However, due to the large lattice mismatch (14.6\%), the growth of InSb on GaAs results in a large number of dislocations at the material interface, reducing the electron mobility. The dislocation density decreases and electron mobility increases significantly for thicker InSb layers. To resolve this problem, it is desirable to grow a highly resistive buffer layer with a lattice constant similar to InSb that will contain the dislocations. A well suited material for this buffer layer is InAlSb due to its similar lattice constant, larger bandgap, and higher resistivity. Recently, improvement of InSb mobility in thin layers has been reported by growth on InAlSb buffers on InP substrates by molecular beam epitaxy (MBE)[6]. We have previously reported the growth of InAlSb layers on $\mathrm{GaAs}$ and $\mathrm{InSb}$ by metal organic chemical vapor deposition (MOCVD) [7]. This report describes the growth by MOCVD of InSb on GaAs substrates with InAlSb buffers.

\section{EXPERIMENTAL TECHNIQUES}

Epitaxial growth was carried out on an EMCORE vertical, high-speed rotating-disk reactor. The sources used for InAlSb were trimethylindium (TMIn), tritertiarybutylaluminum (TTBAl), and triethylantimony (TESb) for In, Al, and Sb, respectively. For InSb layers, we have used TMIn and trisdimethylaminoantimony (TDMASb) as sources for In and Sb. The choice of TDMASb is based on earlier growths where higher InSb electron mobilities were obtained as compared to using TESb $[8,9]$. These choices for sources complicate matters since TTBAl and TDMASb react in the gas phase. To overcome this problem, TTBAl was introduced to the 


\section{DISCLAIMER}

This report was prepared as an account of work sponsored by an agency of the United States Government. Neither the United States Government nor any agency thereof, nor any of their employees, make any warranty, express or implied, or assumes any legal liability or responsibility for the accuracy, completeness, or usefuiness of any information, apparatus, product, or process disclosed, or represents that its use would not infringe privately owned rights. Reference herein to any specific commercial product, process, or service by trade name, trademark, manufacturer, or otherwise does not necessarily constitute or imply its endorsement, recommendation, or favoring by the United States Government or any agency thereof. The views and opinions of authors expressed herein do not necessarily state or reflect those of the United States Government or any agency thereof. 


\section{DISCLAIMER}

Portions of this document may be illegible in electronic image products. Images are produced from the best available original document. 


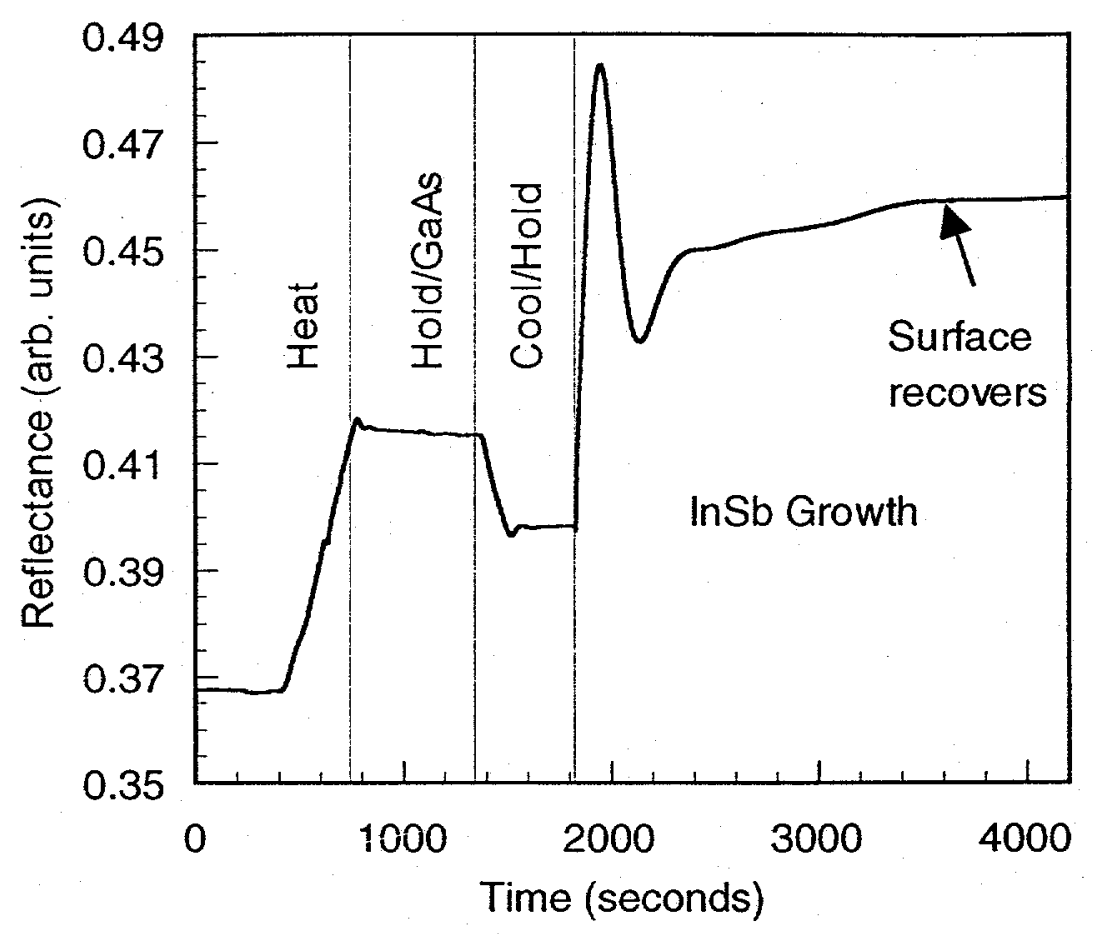

Figure 1: In-situ reflectance monitor during the growth of InSb on GaAs of a sample exhibiting good surface morphology.

reactor in a separate line from the other sources and the reactor was purged with hydrogen ( $>5$ minutes) between the growth of layers containing either TTBAl or TDMASb. Hydrogen was used as the carrier gas at a total flow of $14.5 \mathrm{slpm}$, the susceptor rotation was $1100 \mathrm{rpm}$, and growth was done at 70 Torr. Samples were grown on semi-insulating GaAs consisting of varying compositions of InAlSb layers both with and without an InSb layer on top for comparison of electronic properties and surface morphology. Additional samples of InAlSb were grown on InSb substrates to determine the alloy composition. Prior to $\mathrm{In}(\mathrm{Al}) \mathrm{Sb}$ growth, $100 \mathrm{~nm}$ GaAs buffers were grown at $630^{\circ} \mathrm{C}$ before cooling to growth temperature. In addition, bulk GaAs growths were performed prior to each growth run to replicate growth conditions as best as possible. Without the intermediate GaAs growths, the InSb/InAlSb growths were not repeatable, possibly from chamber conditions or material interaction from the susceptor. The InSb growth temperature, growth rate, and TDMASb/TMIn vapor phase ratio were $400^{\circ} \mathrm{C}, 0.5 \mu \mathrm{m} /$ hour, and 1.0 , respectively. The InAlSb growth temperature, growth rate, and TESb/(TTBAl+TMIn) vapor phase ratio were $440^{\circ} \mathrm{C}, 0.65 \mu \mathrm{m} / \mathrm{hour}$, and 4.0-5.0, respectively. During purges transistioning from InAlSb to InSb growth, a thin cap $(\sim 5 \mathrm{~nm})$ of $\mathrm{InSb}$ using TMIn and TESb was grown prior to the purge and at 1 minute intervals during the purge to passivate the surface.

Growth runs were monitored using in situ reflectance to evaluate surface morphology development[10]. Sample thicknesses were measured using a groove technique. Room temperature Hall measurements using the Van der Pauw technique were used to determine the electron mobility and majority carrier type and concentration. Double crystal $x$-ray diffraction (DCXRD) (004) and (335) reflections were used to determine InAlSb alloy composition by correcting for residual strain in the layers. 


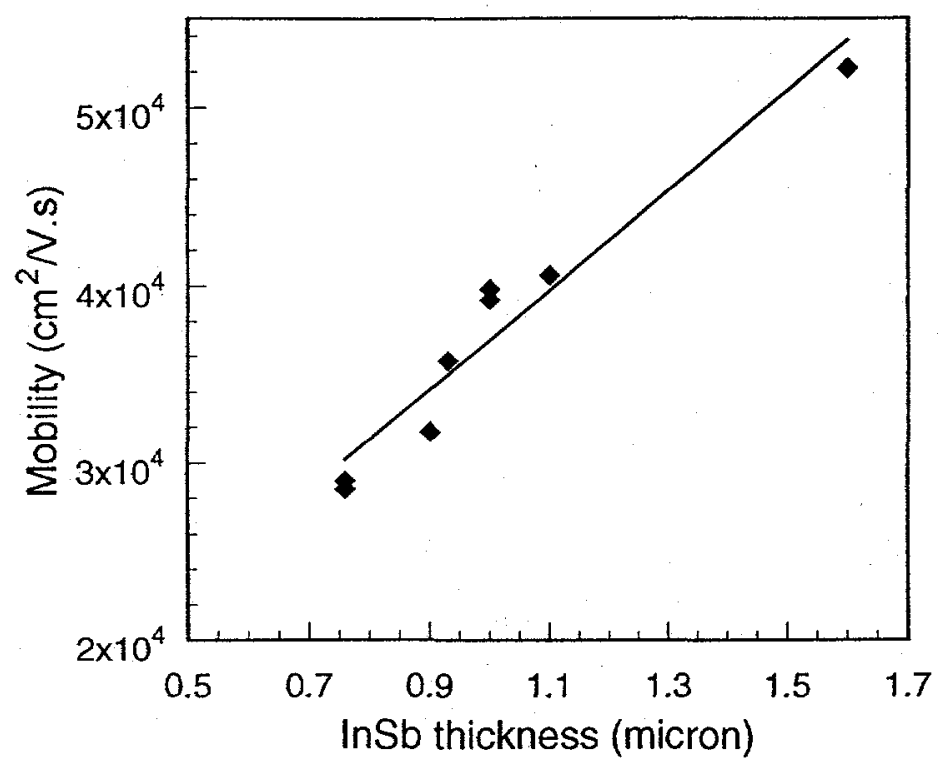

Figure 2: Electron mobility of InSb layers of varying thickness grown on GaAs substrates.

\section{RESULTS AND DISCUSSION}

The growth of InSb on GaAs was examined to optimize growth conditions to ensure good surface morphology and electron mobility. We have used a predeposition of TMIn for 2-5. seconds prior to introducing TDMASb to the reactor, a technique previously reported, to obtain high electron mobility InSb layers[4]. A representative reflectance spectrum of a typical sample growth run is shown in Figure 1. A rise and oscillation in the reflectance signal is observed indicating typical layer growth, followed by a steady increase, indicating an improvement in surface morphology likely due to a decrease in dislocation density. The reflectance signal then flattens out indicating no further improvement in surface morphology. For samples with poor surface morphology due to non-optimal V/III ratios, the reflectance decreases at the start of the InSb growth and never recovers. The electron mobility of the InSb layers was found to be strongly dependent on layer thickness between $0.7-1.6 \mu \mathrm{m}$, as shown in Figure 2, with a nearly linear dependence. High quality InSb growth was achieved as indicated by a $1.6 \mu \mathrm{m}$ thick InSb layer with a measured electron mobility of $52,000 \mathrm{~cm}^{2} / \mathrm{V} \cdot \mathrm{s}$.

For $\operatorname{In}_{1-\mathrm{x}} \mathrm{Al}_{\mathrm{x}} \mathrm{Sb}$ samples grown on InSb substrates, we have measured compositions of $0.002 \leq x \leq 1.0$ which vary with the TTBAl/(TTBAI+TMIn) ratio as shown in Figure 3 [7]. The curves in Figure 3 are drawn as an aid to the eye for determining the trends at the different temperatures. The measured alloy compositions were found to be repeatable from run to run and were applied to the growth of InAISb on GaAs substrates. For the growth of InAlSb on GaAs, the best surface morphologies were obtained by including a predeposition of TMIn and an InSb nucleation layer of $\sim 40 \mathrm{~nm}$. The incorporation of aluminum in the not intentionally doped material resulted in a drastic change in electronic properties, from n-type and high mobility $\mathrm{InSb}$ to $\mathrm{p}$-type and low mobility InAlSb. All $\mathrm{In}_{1-\mathrm{x}} \mathrm{Al}_{\mathrm{x}} \mathrm{Sb}$ samples examined with compositions ranging from $0.05 \leq x \leq 0.25$ were found to be $p$-type with carrier concentrations between $5 \times 10^{16} \mathrm{~cm}^{-3}$ and $2 \times 10^{17} \mathrm{~cm}^{-3}$ and mobilities of less than $900 \mathrm{~cm}^{2} / \mathrm{V} \cdot \mathrm{s}$. For $\mathrm{In}_{1-\mathrm{x}} \mathrm{Al}_{\mathrm{x}} \mathrm{Sb}$ samples with $\mathrm{x}<0.01$, but with a finite aluminum content, Hall measurements indicated n-type material with mobilities greater than $10,000 \mathrm{~cm}^{2} / \mathrm{N} \cdot \mathrm{s}$. This change for very low aluminum content is likely the result of measuring the thin InSb nucleation layer rather than the InAlSb, since the $\operatorname{In}_{1-x} \mathrm{Al}_{x<0.01} S b$ bandgap 


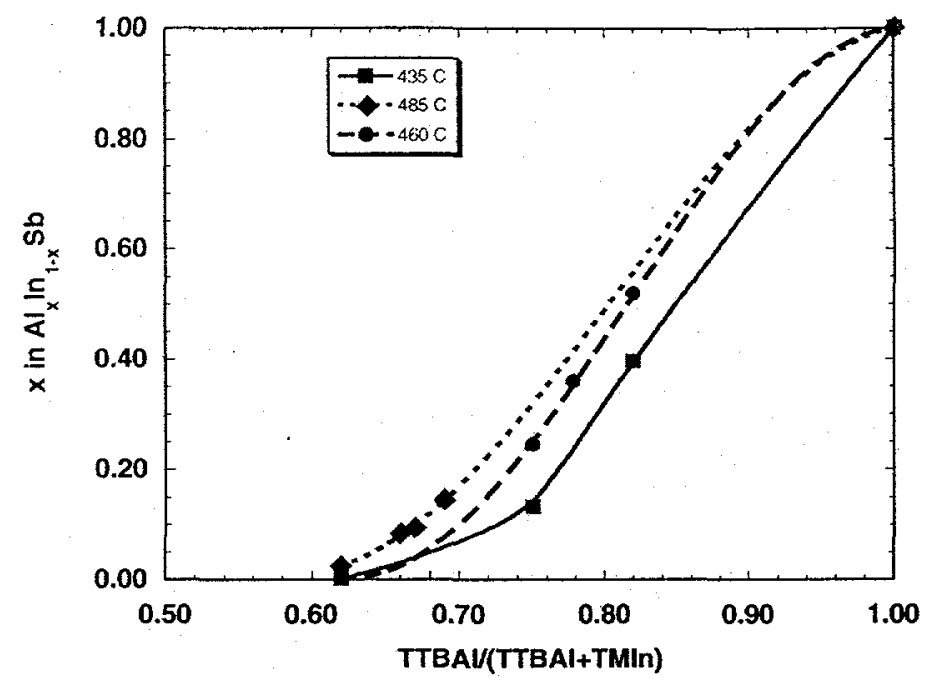

Figure 3: Aluminum composition in InAlSb for varying TTBAl/(TTBAl+TMIn) vapor phase ratio at three growth temperatures.

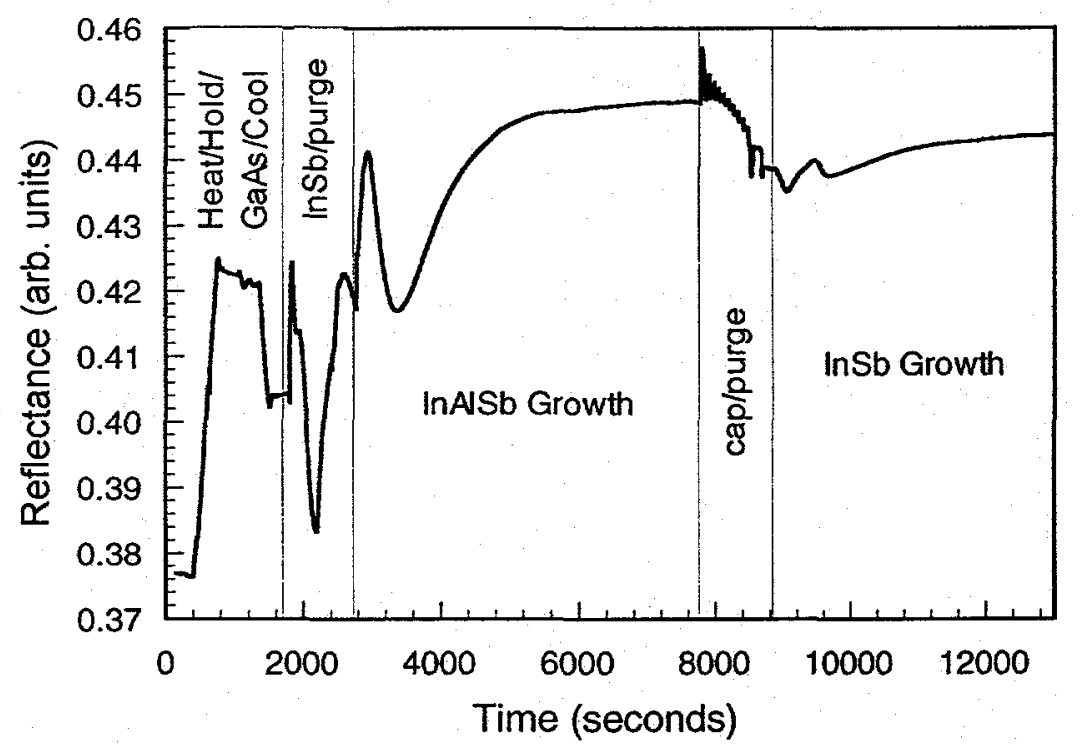

Figure 4: In-situ reflectance spectrum of InSb grown on an $\operatorname{In}_{0.78} \mathrm{Al}_{0.22} \mathrm{Sb}$ buffer and $\mathrm{GaAs}$ substrate.

is not sufficiently different than InSb. The values for resistivity in the $\operatorname{In}_{1-x} \mathrm{Al}_{x} S b$ samples $(0.05 \leq x \leq 0.25)$ were found to be $10-20$ times greater than for InSb. In order to provide the highest buffer layer resistance as required for devices, it is important to keep the buffer layer as thin as possible without compromising the mobility in the active layer.

Samples were grown on GaAs substrates with a top active layer of InSb and an InAlSb buffer with thicknesses of $0.5-0.6 \mu \mathrm{m}$ and $\sim 0.9 \mu \mathrm{m}$, respectively. The thickness of the InAlSb buffer layer was chosen in the interest of keeping the buffer as thin as possible while providing a smooth interface for which to grow the topmost InSb. The reflectance signal provided a good 


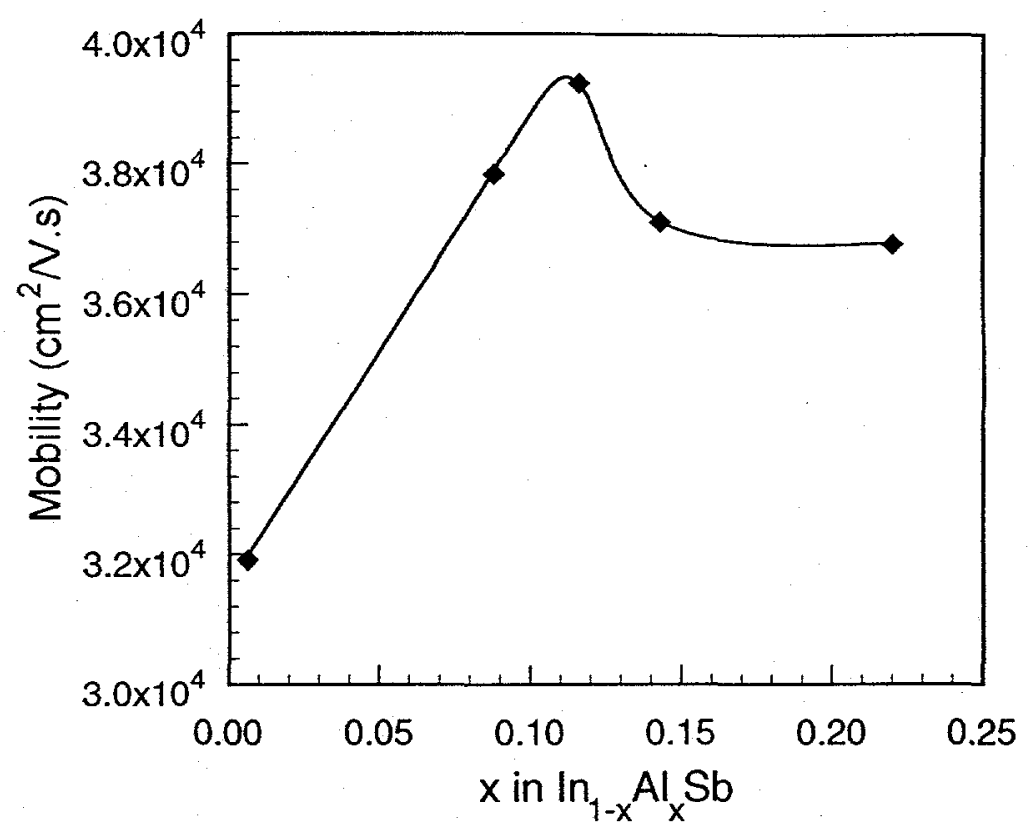

Figure 5: Electron mobility dependence on aluminum composition in the InAlSb buffer of InSb/nAlSb heterostructures grown on GaAs substrates.

indication of the InAlSb surface morphology as it evolved during growth. A reflectance pattern for a sample with an $\operatorname{In}_{0.78} \mathrm{Al}_{0.22} \mathrm{Sb}$ buffer is shown in Figure 4. During the initial growth of InAlSb, a large dip is observed in the reflectance signal indicating a roughening of the surface. At -5600 seconds on the time scale corresponding to $\sim 0.5 \mu \mathrm{m}$ of InAlSb growth, the surface morphology had nearly fully recovered as indicated by a flattening of the reflectance signal in Figure 4 . This surface recovery occurred between $0.5-0.9 \mu \mathrm{m}$ for these samples and seemed to have no correlation with buffer layer composition nor with the resulting electron mobility.

The dependence of electron mobility on $\mathrm{x}$ in the $\mathrm{In}_{1-\mathrm{x}} \mathrm{Al}_{\mathrm{x}} \mathrm{Sb}$ buffer is shown in Figure 5 . The highest electron mobility measured was $>39,000 \mathrm{~cm}^{2} N \cdot \mathrm{s}$ for an $\operatorname{In}_{0.88} \mathrm{Al}_{0.12} \mathrm{Sb}$ buffer and a $0.55 \mu \mathrm{m}$ thick InSb layer. This is a significant improvement over direct InSb growth on GaAs, where an InSb thickness of $\geq 1 \mu \mathrm{m}$ is required to obtain a comparable mobility, as indicated in Figure 2. A reduction in the electron mobility for smaller aluminum compositions $(x<0.12)$ is likely a result of the reduced bandgap of the buffer layer, where the electrons are less confined to the InSb layers. A decrease in electron mobility for increased aluminum composition $(x>0.12)$ is due to the higher lattice mismatch between the InSb and InAlSb buffer, which may result in the formation of dislocation networks at the InSb interface. These explanations require further experimental evidence and statistics for support, but we believe they are valid conclusions nonetheless.

\section{CONCLUSION}

High electron mobility InSb has been grown by MOCVD on GaAs substrates with a strong dependence on layer thickness. Through use of an insulating InAlSb buffer layer, high mobility InSb can be grown with a smaller layer thickness, as is desirable for magnetoresistive sensor applications. An electron mobility of $\sim 40,000 \mathrm{~cm}^{2} / \mathrm{V} \cdot \mathrm{s}$ was measured for a $0.55 \mu \mathrm{m}$ thick InSb layer with an $\mathrm{In}_{0.88} \mathrm{Al}_{0.12} \mathrm{Sb}$ buffer layer, where a thickness of $\sim 1 \mu \mathrm{m}$ is required for direct 
growth on GaAs to get a comparable mobility. Varying the aluminum composition in the $\mathrm{In}_{1-}$ ${ }_{x} \mathrm{Al}_{x} \mathrm{Sb}$ buffer resulted in an increase in the measured mobility for $\mathrm{x}<0.12$ due to the InAlSb bandgap increase, and a decrease in mobility for $x>0.12$ due to an increase in the InSb/nAlSb lattice mismatch.

Sandia is a multiprogram laboratory operated by Sandia Corporation, a Lockheed Martin Company, for the United States Department of Energy under contract DE-AC04-94AL85000.

\section{REFERENCES}

1. J. Heremans, D. L. Partin, C. M. Thrush, and L. Green, Semicond. Sci. Technol. 8 (1993) S424.

2. R. M. Biefeld and G. A. Hebner, J. Crystal Growth 109 (1991) 272.

3. M. A. McKee, B. S. Yoo, and R. A. Stall, J. Crystal Growth 124 (1992) 286.

4. D. L. Partin, L. Green, and J. Heremans, J. Electronic Materials 23 (1994) 75.

5. http://www.emcore.com/devices/pegasus.html

6. D. L. Partin, J. Heremans, and C. M. Thrush, J. Vac. Sci. Technol. B 17 (1999) 1267.

7. R. M. Biefeld, A. A. Allerman, and K. C. Baucom, J. Electronic Materials 27 (1998) L43.

8. K. C. Baucom and R. M. Biefeld, Appl. Phys. Lett. 64 (1994) 3021.

9. D. L. Partin, M. Pelczynski, P. Cooke, L. Green, J. Heremans, and C. M. Thrush, J. Crystal Growth 195 (1998) 378.

[10] W. Breiland, H. Ho, H. Chui, and B. Hammons, J. Crystal Growth 174 (1997) 564. 